BLAKE, H., 2012. Commissioned commentary on: Tai Chi intervention for older adults using assistive device in a senior living community- a pilot study. International Journal of Therapy and Rehabilitation. 19(3), 143.

There is a drive in public health policy to increase physical activity in older age, and to encourage 'active ageing' with the aim of reducing age-related mortality and morbidity. Despite the known physiological and psychosocial benefits of physical activity, inactivity increases with age and there is a need to identify physical activities which can be safely practised in this population.

Over recent years, there has been an increasing interest in Tai Chi as a mechanism to support physical and emotional health in older age. There has also been an exponential increase in the research on age-related health problems such as falling, associated complications including fractures, long-term disability and loss of independence, and the rising medical and social care costs associated with this.

We know that exercise for healthy ageing ideally should include a combination of aerobic, strengthening, and flexibility exercises. The potential benefits of Tai Chi are increasingly being recognized as this co-ordination exercise has low velocity and impact and as such has been demonstrated as appropriate for many older people. The authors have highlighted that there are a proportion of older adults relying on assistive ambulatory devices (AADs) for daily mobility, and suggest that these individuals are at increased risk of falls related to their dependence on the device, increased postural sway, altered gait and increased fear of falls. The authors propose that this is the first exercise 
programme specifically designed for older AAD users to improve balance and strength at a standing position.

\section{This study}

In this small, uncontrolled pilot study, the authors have investigated the outcomes of a 6form Tai Chi (TC) programme provided to older adults using assistive ambulatory devices (AADs). The intervention consisted of three 60-minute sessions of Tai Chi per week, delivered over a 12-week period. The group constituted of both independently and non-independently living residents of a senior living community. In addition to assessing fear of falling, the authors used objective measures before and after the intervention, to assess sit-to-stand, balance, and gait ability in order to determine an older person's risk for falls. While only 13 participants completed the programme, the findings suggest that there may be potential for improvement in balance and a reduction in fear of falling in this population, although there were no clear indications in this small sample that lower extremity strength was improved.

While it is known that balance can be improved in older adults with Tai Chi intervention, this has not previously been investigated specifically in those older adults who are AAD users yet these individuals may have specific needs. The psychological outcomes of the participants in this study were not assessed although it has recently been reported that Tai Chi may impact upon self-efficacy whereas conventional physiotherapy does not (Tousignant et al, 2012). This warrants investigation, since self-efficacy may serve to reduce fear of falls, and may have some bearing on adherence with, and drop-out from, physical activity programmes. This pilot study suggests that TC may be an appropriate 
physical activity to reduce risk of falls in AAD users, although this needs to be tested in a large-scale randomized controlled trial with longer follow-up periods.

\section{Conclusions}

Regular exercise should be a fundamental aspect of caring for older people who are living in the community. Tai Chi may be particularly suitable for older people and the published literature proposes that Tai Chi may increase muscle strength and improve both balance and concentration. This pilot study provides a useful insight into the potential for delivery of such interventions with older adults using AADs, and raises some interesting issues, which should be considered in the design of future trials. This study also supports the findings of recent reviews, which have recognized the benefits of Tai Chi for improving balance with the aim of reducing falls (Leung et al, 2011; Blake and Hawley, 2011).

\section{References}

Blake H, Hawley H (2011) Effects of Tai Chi Exercise on Physical and Psychological Health of Older People. Curr Aging Sci 5(1): 19-27

Leung DP, Chan CK, Tsang HW, Tsang WW, Jones AY (2011) Tai chi as an intervention to improve balance and reduce falls in older adults: A systematic and metaanalytical review. Altern Ther Health Med 17(1): 40-8

Tousignant M, Corriveau H, Roy PM, Desrosiers J, Dubuc N, Hébert R et al (2012) The effect of supervised Tai Chi intervention compared to a physiotherapy program on fallrelated clinical outcomes: a randomized clinical trial. Disabil Rehabil 34(3): 196-201 